Check for updates

Cite this: RSC Adv., 2018, 8, 38363

Received 24th September 2018 Accepted 24th October 2018

DOI: 10.1039/c8ra07913g

rsc.li/rsc-advances

\section{Superhydrophobic engineering materials provide a rapid and simple route for highly efficient self- driven crude oil spill cleanup $\dagger$}

\author{
Hongbo Xu, (D) a Shulong Bao, ${ }^{b}$ Liuting Gong, ${ }^{a}$ Renping Ma, ${ }^{d}$ Lei Pan, ${ }^{a}$ Yao Li ${ }^{\star c}$ \\ and Jiupeng Zhao*a
}

Traditional superhydrophobic material use depends on two processes: creating a rough structure on a material surface and modifying the rough surface with low surface energy materials. However, common preparation methods are time-consuming, complex and cost-ineffective. Furthermore, these methods usually rely on chemicals, and evidently that will restrict mass preparation and application of superhydrophobic materials. This study reports a simple polypropylene (PP) solution-based process for producing PP hierarchical structures on commercial copper mesh (low surface energy materials), without modifying the low surface energy materials. The hierarchical structures of copper meshes, surface modified with PP, can be rationally controlled by optimizing the PP concentration. The obtained copper mesh showed contact and rolling off angles of $162^{\circ}$ and $7^{\circ}$, respectively. Importantly, no significant performance loss was observed after the superhydrophobic copper meshes were continuously and drastically rinsed with $3.5 \mathrm{wt} \% \mathrm{NaCl}$ solution, or repeated tearing with an adhesive tape for more than 30 cycles, indicating its good durability. After surface modification with PP particles, the copper mesh exhibits both excellent superhydrophobicity and superoleophilicity. Additionally, the asprepared copper mesh can self-float on water surface when deformed into a "miniature boat" shape. Meanwhile, self-driven spilled oil cleanup was achieved using a superhydrophobic copper mesh-formed miniature boat. The miniature boat can realize energy conservation as well as high efficiency. The cleanup rate of the boat is as high as $97.1 \%$, demonstrating its great potential in environmental remediation applications.

\section{Introduction}

With the increasing consumption of crude oil and its derivatives, oil spills during production and transportation happen frequently, ${ }^{\mathbf{1} 2}$ which lead to a great loss of energy, severe environmental pollution and consequent ecological problems. ${ }^{3-5}$ Conventional methods including centrifuges, oil skimmers, depth filters, flotation and sedimentation are useful for immiscible oil/water mixtures separation, but holdbacks such as low efficiency of separation and high energy consumption severely restrict their application. ${ }^{6}$

\footnotetext{
${ }^{a}$ MIIT Key Laboratory of Critical Materials Technology for New Energy Conversion and Storage, School of Chemistry and Chemical Engineering, Harbin Institute of Technology, 150001, Harbin, China. E-mail: jpzhao@hit.edu.cn; Fax: +86 451 86403767; Tel: +8645186403767

${ }^{b}$ Beijing Institute of Space Mechanics and Electricity, 100094, Beijing, China

${ }^{c}$ Center for Composite Material, Harbin Institute of Technology, 150001, Harbin, China.E-mail: Yaoli@hit.edu.cn

${ }^{d}$ Beijing Vocational College of Labour and Social Security, 102200, Beijing, China

$\dagger$ Electronic supplementary information (ESI) available. See DOI: 10.1039/c8ra07913g
}

Combined with superhydrophobicity and oleophilicity, materials can exhibit certain potential to separate oil/water mixtures. ${ }^{7,8}$ When applied to a surface, the wetting behaviour of water from sheeting and complete wetting on superhydrophilic surfaces to spherical droplet formation on superhydrophobic surfaces give inspiration to obtain solution for oil/ water separation. ${ }^{9-17}$ Due to their efficient ability in selective absorption/separation, and favourable recyclability, superhydrophobic materials with porous structures, such as sponges, inorganic or metal fabrics and meshes, have gained broad attention for oil-water separation. ${ }^{18-24}$ Oil would sheet over the surface of these materials, whereas water will form droplets rendering these materials capable of separating water/oil mixtures. ${ }^{25,26}$ The merit of superhydrophobic meshes in the separation of water/oil mixtures is that they allow oils to go through their pores while repressing water on the surface. ${ }^{27,28}$

Currently, many methods have been demonstrated and a number of techniques have been exploited to produce superhydrophobic surfaces on numerous substrates including the template method, ${ }^{29}$ sol-gel process, ${ }^{30}$ self-assembly technique, ${ }^{31,32}$ electro-deposition, ${ }^{33}$ electro-spinning, ${ }^{34}$ hydrothermal synthesis, ${ }^{35}$ chemical vapour deposition, ${ }^{36}$ spray 
coating $^{37}$ and so on. Materials fabricated using the abovementioned methods can realize oil/water separation efficiently, however, the production processes that rely on specific equipment, are commonly time-consuming and costineffective. Furthermore, they require chemicals including strong acid, strong alkali, and expensive reagents (with low surface energy) such as alkyl polysilane, alkyl sulfhydryl, and fluorosubstituted alkane. These requirements severely impede large-scale production and application. Thus, it is highly demanding to develop facile, inexpensive, and environmentally friendly strategies for producing functional absorbent materials. Nowadays, owing to low cost, low density, fascinating flexibility, high mechanical stability and other advantages, metal meshes have been considered as next generation potential material in oil/water separation. ${ }^{38-40}$ In particular, superhydrophobic metal meshes have gained a hot spot for the following reasons: the mesh can work as a filter and provide sufficient room for separating oil/water mixtures, meanwhile, absorbent materials on the mesh can selectively absorb the oil. However, time-consumption, complex operation and costineffectiveness are main holdbacks in preparation processes such as hydrothermal treatment, oxygen plasma pre-treatment, and vapour phase deposition. Thus, pursuing a facile and timesaving method to produce functional absorbent materials for oil/water separation and spilled oil cleanup is evidently of great importance. ${ }^{\mathbf{4 1}-44}$

In our study, a novel superhydrophobic copper mesh-based separation system was developed for the cleanup of water and crude oil spills. The fabrication process only needs several seconds with simple ingredients at room temperature, which makes it not only economical and universal but also timesaving. Specifically, copper meshes with a series of pore diameters were coated with rough PP layers. The present method shows the advantages in many aspects such as facile operation, low costs, short production times, and environmental safety. Thus, this method can be well adopted to fabricate desired PP hierarchical structures on copper mesh under mild conditions. Water contact and rolling off angles of around $162^{\circ}$ and $7^{\circ}$, respectively, were realized on the as-prepared copper meshes. Moreover, after continuous rinsing with $3.5 \mathrm{wt} \% \mathrm{NaCl}$ solution or repeated tearing with an adhesive tape for more than 30 cycles, the copper meshes retained good superhydrophobic performances. The miniature boat by superhydrophobic copper meshes can self-float on water surfaces and automatically recycle crude oil spills with a cleanup rate of up to $97.1 \%$.

\section{Experimental section}

\subsection{Materials}

All solvents and chemicals were of reagent quality and were used without further purification. Hydrochloric acid ( $\mathrm{HCl})$, nitric acid $\left(\mathrm{HNO}_{3}\right)$, acetone, anhydrous ethanol, xylene, sodium chloride $(\mathrm{NaCl})$ and sodium hydroxide $(\mathrm{NaOH})$ were purchased from Sinopharm Chemical Reagent Beijing Co., Ltd. (China). The crude oil was provided by the Chinese Petroleum Corporation. All chemicals described in the experimental section were analytical reagents. PP was purchased from Sigma-Aldrich and copper meshes were obtained from Pengda Business Company. The diameters of the copper meshes were $43 \mu \mathrm{m}$ (350 mesh), 83 $\mu \mathrm{m}$ (180 mesh), $125 \mu \mathrm{m}$ (120 mesh), $187 \mu \mathrm{m}$ (80 mesh), $250 \mu \mathrm{m}$ (60 mesh), and $500 \mu \mathrm{m}$ (30 mesh).

\subsection{Fabrication of a superhydrophobic and oleophilic copper mesh}

The copper mesh (size $5 \mathrm{~cm} \times 5 \mathrm{~cm}$ ) was cleaned ultrasonically in acetone, ethanol, water and $0.05 \mathrm{~mol} \mathrm{l}^{-1} \mathrm{HNO}_{3}$ solution sequentially. This procedure thoroughly removed surface impurities and enhanced the adhesion between the copper mesh and the PP films. As shown in Fig. 1, different amounts of PP (1.5 g, 2 g, $2.5 \mathrm{~g}, 3 \mathrm{~g}, 3.5 \mathrm{~g})$ were added to xylene (100 ml) and stirred for $12 \mathrm{~h}$ at $130{ }^{\circ} \mathrm{C}$. The cleaned copper mesh was immersed in PP solution to deposit PP hierarchical structures. The resultant copper mesh films were washed in acetone and dried in nitrogen to achieve superhydrophobicity and oleophilicity.

\subsection{Measurement of stability of the superhydrophobic copper mesh}

The chemical and mechanical stability of the superhydrophobic copper mesh was evaluated by a continuous rinsing test and a repeated tape tearing test. The manufactured superhydrophobic copper mesh was fixed on a beaker wall, and the beaker was filled with $3.5 \mathrm{wt} \% \mathrm{NaCl}$ solution. Then, the magnetic stirrer was placed in the beaker, and the copper mesh was continuously rinsed at $1500 \mathrm{rpm}$ for different durations. The water contact angle of the copper mesh was recorded accordingly. In addition, the copper mesh was transferred to an adhesive tape and then torn with tape. This process was repeated, and the corresponding water contact angle was recorded after each tearing cycle.

\subsection{Fabrication of a superhydrophobic and oleophilic "copper mesh miniature boat" for self-driven oil crude collection}

As shown in Fig. 1, a copper mesh film, $5.0 \mathrm{~cm} \times 5.0 \mathrm{~cm}$ in size, was immersed in xylene. The resultant copper mesh was then

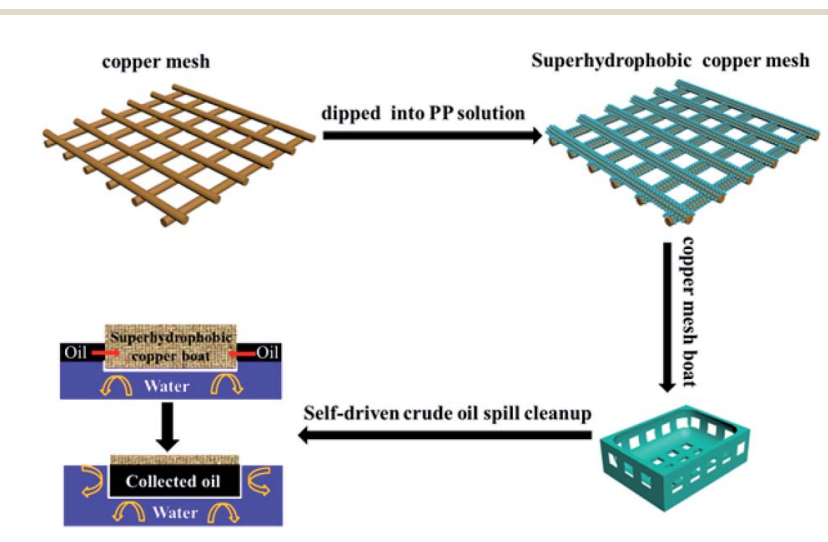

Fig. 1 Schematic illustration of the fabrication of a superhydrophobic copper mesh with PP and a superhydrophobic boat made from the copper mesh for self-driven oil spill cleanup. 
modified with PP to gain superhydrophobicity and oleophilicity. The superhydrophobic copper mesh was folded into the "miniature boat" form (size $3.4 \mathrm{~cm} \times 3.4 \mathrm{~cm} \times 0.8 \mathrm{~cm}$ ). The "copper mesh miniature boat" showed excellent superhydrophobic and oleophilic properties. The superhydrophobic stability of the "miniature boat" was evaluated by measuring the cleanup weight, which reflects its long-term coating ability. For the crude oil cleanup experiment, $30 \mathrm{~g}$ crude oil was added to a beaker with water inside to simulate a crude oil spill. Then, the miniature boat was placed in the beaker for self-driven oil spill cleanup. The cleanup rate (CR) was calculated using the following eqn (1):

$$
\mathrm{CR}=\frac{W_{\mathrm{a}}}{W_{\mathrm{o}}}
$$

where $W_{\mathrm{o}}, W_{\mathrm{a}}$ are the weights of the crude oil and the oil collected by the miniature boat, respectively.

\subsection{Characterizations of as-prepared copper meshes}

The surface morphologies were characterized using scanning electron microscopy (SEM, SUPRA-55, ZEISS, Germany). The original and PP-decorated copper meshes were sputtered with gold (thickness of $5 \mathrm{~nm}$ ). Water contact angles were measured at room temperature by a high speed digital camera (JY-PHa 2012D2, Beijing Dingsheng Digital Technology Equipment Co., Ltd., Beijing, China), and more than five repeated measurements were conducted for each sample. For the water sliding angle measurements, a $5 \mu$ l liquid droplet was used. Additional details of this measurement process can be found in the previous study. ${ }^{41}$

\section{Results and discussion}

Fig. 1 is the schematic illustration of the preparation of the superhydrophobic copper mesh with PP and the fabrication of a superhydrophobic boat derived from the copper mesh for selfdriven oil spill collection and cleanup. First, the PP polymer was coated on the copper mesh via immersion and deposition in xylene solution at a high temperature (about $130{ }^{\circ} \mathrm{C}$ ), where the formation of PP nanoparticles is completed within $10 \mathrm{~min}$. After drying, the mesh is exposed to air at room temperature (about $25{ }^{\circ} \mathrm{C}$ ). As it cools, the mesh surface gradually changes from brown, the colour of copper, to light white, which indicates that PP nanoparticles have been generated and adhered to the strings of copper mesh. Several advantages can be seen in this approach. The whole process is completed in xylene solution with PP in the absence of chemicals with low surface free energy such as alkyl polysilane, alkyl sulfhydryl, or fluorosubstituted alkane, which indicates lower costs. Furthermore, the route is facile and no complex techniques or special equipments are involved; thus, industrial mass manufacture of superhydrophobic nanostructures on copper mesh surface is highly practical. Self-driven oil spill cleanup was also achieved using a miniature boat formed by superhydrophobic copper mesh. The miniature boat could realize energy conservation as well as high efficiency.
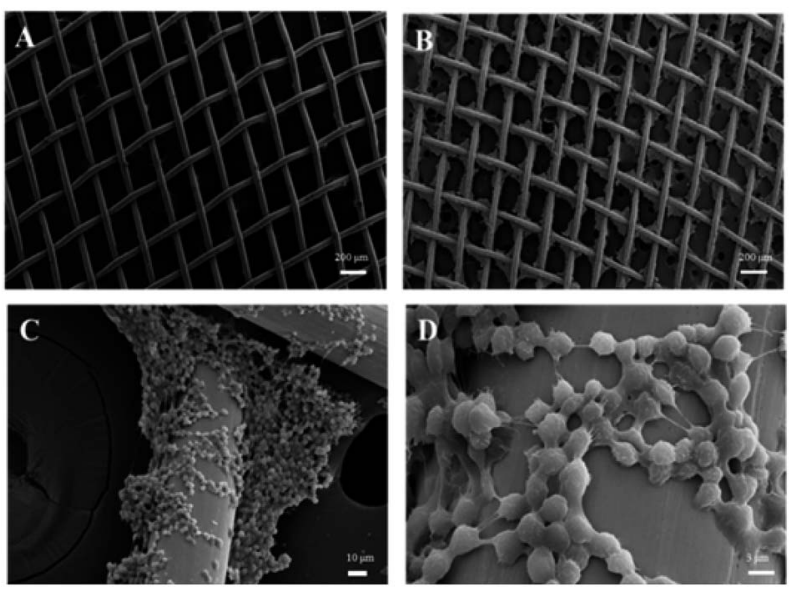

Fig. 2 (A) SEM image of the pristine copper mesh; (B-D) SEM image and high-magnification SEM image of PP hierarchical structures (prepared with PP $25 \mathrm{mg} \mathrm{ml}^{-1}$ ) on copper mesh.

SEM images were used to investigate the morphology of the copper meshes before and after immersion in the PP solution (shown in Fig. 2A and B). The pristine copper mesh with no other treatment has a smooth surface (Fig. 2A). After immersion in PP solution ( $25 \mathrm{mg} \mathrm{ml}^{-1}$ ) at $30^{\circ} \mathrm{C}$ for $10 \mathrm{~min}$, large amount of regular and hierarchical structures are generated on the surface of the copper mesh (Fig. 2B). The corresponding high magnification SEM image illustrates that the hierarchical structure is formed by nanoparticles with an average size of about $200 \mathrm{~nm}$ (Fig. 2C and D), and most of the nanoparticles aggregate into microparticles of about $4 \mu \mathrm{m}$. With this mode of aggregation, the nanoparticles, microparticles and micrometer-sized copper wires form a hierarchical architecture with dual-scale roughness, which enhances the superhydrophobicity of the copper mesh.

All the PP-decorated copper meshes displayed rough surface microstructures at different PP concentrations similar to the $25 \mathrm{mg} \mathrm{ml}{ }^{-1}$ PP-decorated copper meshes (Fig. S1 $\dagger$ ); however, these PP-decorated copper meshes showed different levels of wettability. Fig. S2† shows CA for copper meshes of 350, 180, $120,80,60$, and 30 mesh counts with different PP concentrations. Evidently, all the PP-decorated copper meshes showed hydrophobicity, as the CAs were above $130^{\circ}$. The CAs of the PPdecorated copper meshes can be controlled by varying the PP concentration and mesh count. It can be seen that the CAs are all above $150^{\circ}$ when the PP concentration is higher than $25 \mathrm{mg}$ $\mathrm{ml}^{-1}$. In order to assure the uniformity of copper mesh diameters and lower the cost, the optimal concentration is $25 \mathrm{mg}$ $\mathrm{ml}^{-1}$. Fig. S3A-F† shows the SEM images of copper mesh structures obtained with PP concentration of $25 \mathrm{mg} \mathrm{ml}^{-1}$ and with different mesh numbers, which indicates that they have similar structure with that in Fig. 1B.

Due to its excellent superhydrophobicity (WCA of about $160^{\circ}$ ), copper mesh-120 was chosen for further investigation. The digital photographs of water droplets and an oil droplet on the superhydrophobic copper mesh are displayed in Fig. 3A and $\mathrm{B}$, respectively. Water droplets can stably remain on the surface of the boat-shaped copper mesh and maintain a spherical 


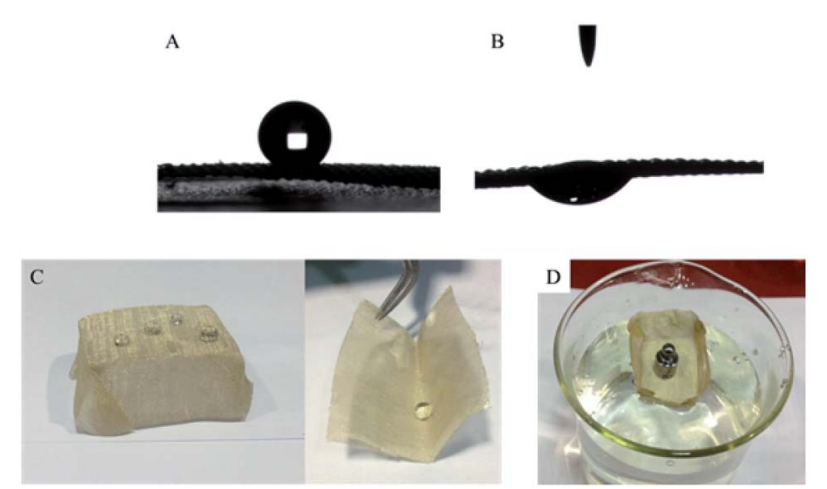

Fig. 3 Photographs of the water droplet with a contact angle of (162.0 $\pm 1.4)^{\circ}$ (A) and an oil droplet with a CA of nearly zero (B) on asprepared superhydrophobic and superoleophilic material; (C) water CA of a superhydrophobic copper mesh at different places. (D) Oblique view of the long-term flotation of the superhydrophobic miniature boat loaded with four nuts $(15 \mathrm{~g})$.

shape, while the oil droplets spread rapidly. The photograph (shown in Fig. 3C) clearly shows the wetting behavior of the water droplets on the superhydrophobic miniature boat. Interestingly, the superhydrophobic miniature boat can still float freely on water when loaded with one weight $(20 \mathrm{~g})$, indicating its outstanding superhydrophobicity (Fig. 3D). When different materials were applied on the substrate, PP hierarchical structures with different sizes and height were fabricated, which exhibits an admirable super-hydrophobic performance. For instance, the water droplets can stably remain on the surface of a stainless steel mesh with PP having a spherical shape. This displays the excellent super-hydrophobic performance of steel mesh with PP. The spherical-shaped water droplets was also obtained on other material surfaces with PP (see Fig. S4†). This demonstrated that the method can be widely applied to a variety of materials, fabricating super-hydrophobic surfaces. The stability of the superhydrophobic copper mesh was evaluated using $3.5 \mathrm{wt} \% \mathrm{NaCl}$ solution to simulate ocean water, to test its

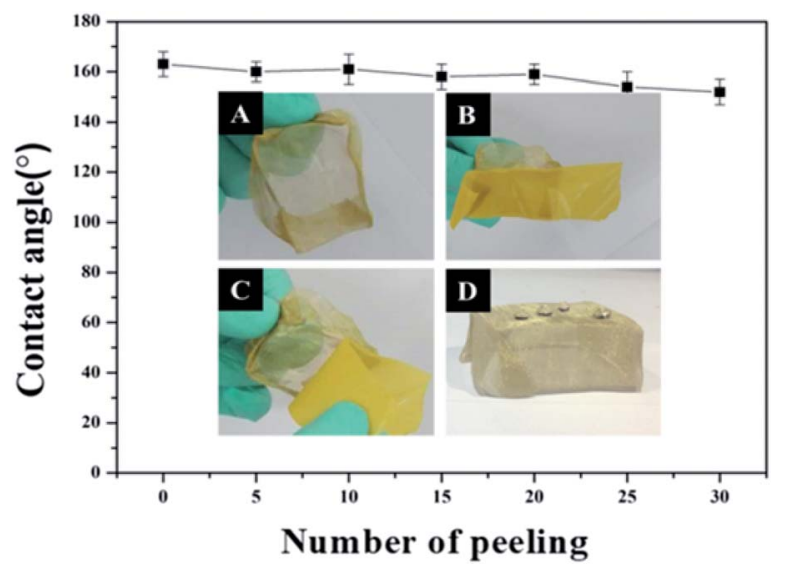

Fig. 4 Water CA of superhydrophobic fabric repeatedly torn by adhesive tape. The inset depicts the tearing process. (A) A miniature boat; (B) the miniature boat with an adhesive tape, (C) the miniature boat with the adhesive tape peeled off; (D) water droplets on the mini boat after peeling off the adhesive tape.

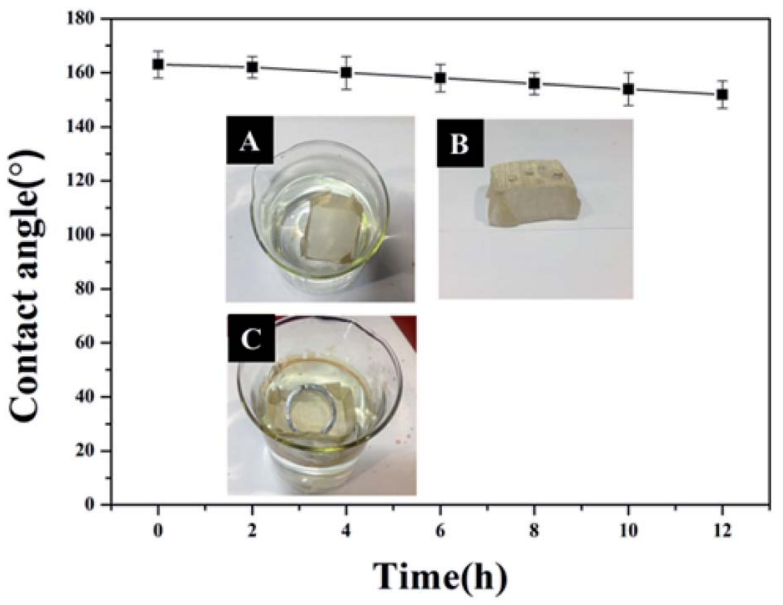

Fig. 5 Water contact angles of a superhydrophobic miniature boat continuously rinsed with $3.5 \mathrm{wt} \% \mathrm{NaCl}$ solution for different durations. The inset depicts the process of the rinse.

water resistance under continuous rinsing and an adhesive tape to simulate external force, which will test its force resistance under repeated tearing. After rinsing for a long time of up to $8 \mathrm{~h}$ (Fig. 4) or a 30-cycle tear test with adhesive tape (Fig. 5), the water CA remained above $150^{\circ}$. The slight decline indicates that the material possesses outstanding stability. Fig. 6 displays the dynamic CA measurements on superhydrophobic copper mesh modified with $\mathrm{PP}$ at $\mathrm{pH}$ values from 1 to 14; insets are corresponding photographs of the water CA. This superhydrophobic copper mesh, which has excellent stability, could provide promising materials for tackling environmental and energy issues such as crude oil spill cleanup.

Although tremendous efforts have been put into preparation of superhydrophobic materials and fabrication of devices for oil spill cleanup, extra power input is still necessary to achieve the continuous separation of oil and water. In order to achieve an automatic collection of oil without any power input, we propose the use of a miniature superhydrophobic boat, as superhydrophobic porous materials can float freely on water. As shown in Fig. 7A, a miniature boat is fabricated from copper

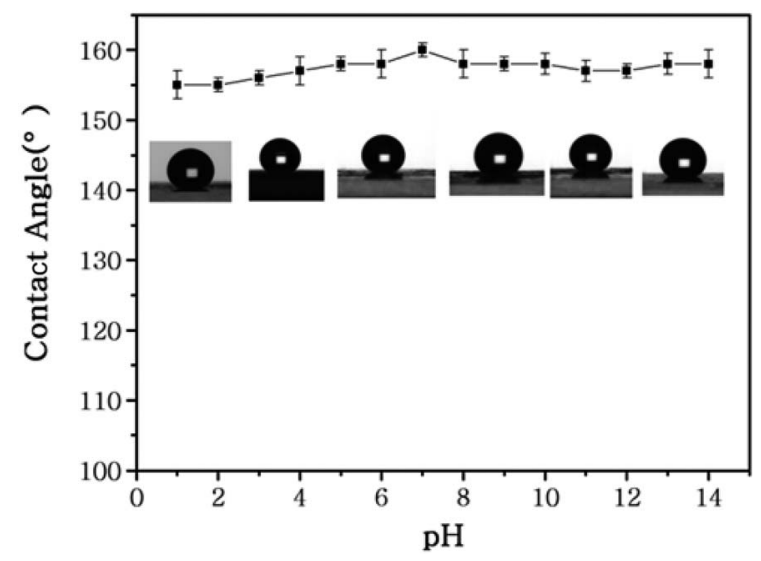

Fig. 6 Water contact angles of a superhydrophobic miniature boat at different $\mathrm{pH}$ values. The insets show the corresponding photographs of the water $\mathrm{CA}$. 

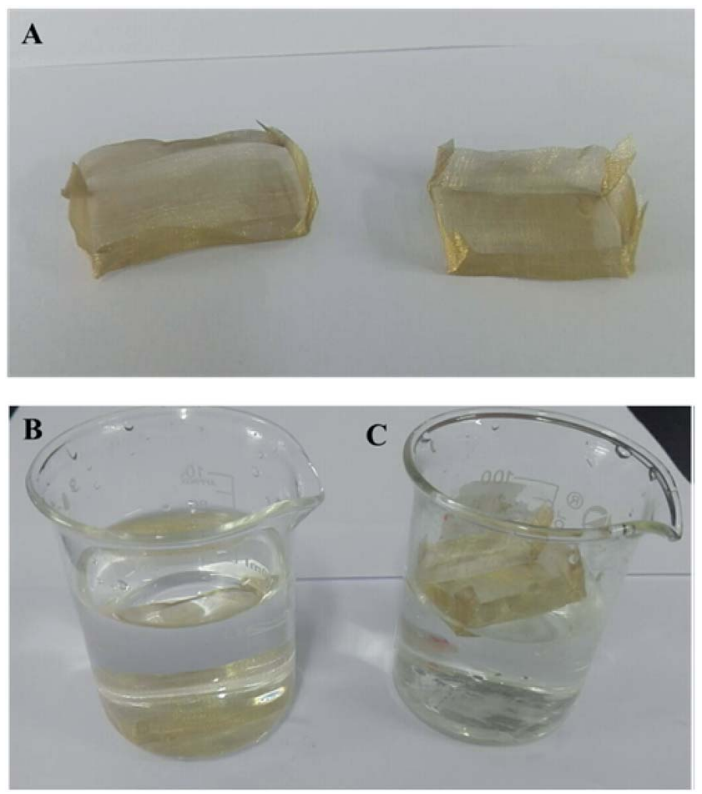

Fig. 7 (A) Photograph of a miniature boat fabricated from copper mesh and superhydrophobic copper mesh; (B) a miniature boat fabricated from pristine copper mesh sinks in water; (C) a miniature boat fabricated from superhydrophobic copper mesh floats in water.
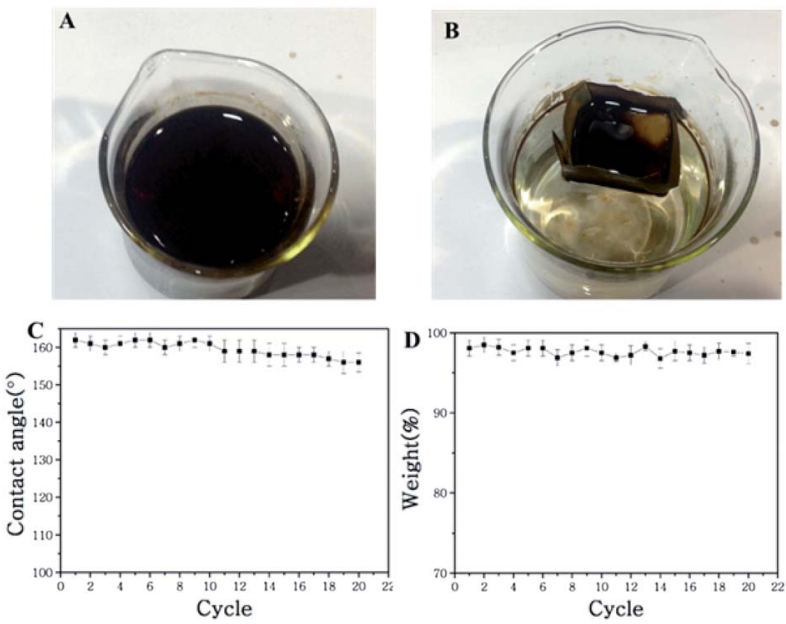

Fig. 8 (A and B) The process of self-driven crude oil spill cleanup of diesel oil from water using the as-prepared miniature boat; (C) water CAs of a reused miniature boat; (D) the crude oil cleanup rate of the reused miniature boat.

mesh and superhydrophobic copper mesh. The miniature boat fabricated from pristine copper mesh cannot float on water (Fig. 7B), while the miniature boat made from superhydrophobic copper mesh can float freely on water (Fig. 7C).

Fig. 8 illustrates the process of a self-driven crude oil spill cleanup simulation. The water is covered with crude oil spill (30 $\mathrm{g}$ ), and the miniature boat was placed the surface of water. Under permeation, the crude oil spill was absorbed and entered the miniature boat. Meanwhile, there was no observation of water permeation into the boat (Fig. 8B). In less than 1 minute, the oil absorption process was complete, and about $29.1 \mathrm{~g}$ of oil was collected. The crude oil clean-up rate reached as high as $97.1 \%$. Importantly, the mini boat from the superhydrophobic copper mesh can be reused for self-driven oil spill cleanup. The reused miniature boat exhibited a water CA of about $152^{\circ}$, which is slightly less than the original $162^{\circ}$, and the crude oil cleanup rate reached as high as $95 \%$ after 30 times of usage (shown in Fig. 8C and D). Meanwhile, the digital photograph (shown in Fig. S5 $\dagger$ ) clearly displays the water droplets maintaining a spherical shape on the surface of the reused miniature boat, which indicates that the copper mesh still possesses superhydrophobicity. Compared to pristine copper mesh, the CA and weight-bearing capacity of the reused copper mesh has slightly decreased, but the mesh is still capable of floating and separating oil and water (shown in Fig. S5 $\dagger$ ). This demonstrates that the present approach exhibits excellent stability and holds highly promising application in oil/water separation and offshore crude oil spill cleanup.

\section{Conclusions}

In summary, we report a facile approach to fabricate robust superhydrophobic copper meshes through one-step PP solution-immersion process. This copper mesh exhibited excellent oil/water separation properties. The morphology of the PP coatings on copper meshes was mainly affected by the concentration of $\mathrm{PP} / x y l e n e$ solutions. Water contact and rolling off angles of $162^{\circ}$ and $7^{\circ}$, respectively, were realized on the asprepared copper meshes. Moreover, the superhydrophobic copper meshes can withstand continuous and drastic rinsing with $3.5 \mathrm{wt} \% \mathrm{NaCl}$ solution and repeated tearing with an adhesive tape for more than 30 cycles. The results indicate that the superhydrophobic copper meshes have good mechanical stability and excellent resistance to water and external force. The as-prepared copper meshes exhibit superwetting properties, excellent mechanical durability and self-driven crude oil spill cleanup capabilities. Furthermore, the superhydrophobic boat by copper meshes can automatically recycle crude oil spills while floating freely on water with a cleanup rate of up to $97.1 \%$, indicating great potential in environmental remediation applications. Considering the simple fabrication process, high commercial availability of copper mesh, excellent reusability, as well as good mechanical stability, this method holds highly promising application in oil/water separation and off shore crude oil spill cleanup.

\section{Conflicts of interest}

There are no conflicts to declare.

\section{Acknowledgements}

This study was supported by the National Natural Science Foundation of China (No. 51572058, 51502057, 51702068), China Postdoctoral Science Foundation (No. 2016M601427), the Natural Science Foundation of Heilongjiang Province (E201436), National Key Research \& Development Program (2016YFB0303903, 2016YFE0201600), the International Science 
\& Technology Cooperation Program of China (2013DFR10630, 2015DFE52770), and Foundation of Equipment Development Department (6220914010901).

\section{Notes and references}

1 B. Wang, W. Liang, Z. Guo and W. Liu, Biomimetic superlyophobic and super-lyophilic materials applied for oil/ water separation: a new strategy beyond nature, Chem. Soc. Rev., 2015, 44, 336-361.

2 Z. X. Xue, Y. Z. Cao, N. Liu, L. Feng and L. Jiang, Special wettable materials for oil/water separation, J. Mater. Chem. A, 2014, 2, 2445-2460.

3 A. K. Kota, G. Kwon, W. Choi, J. M. Mabry and A. Tuteja, Hygro-responsive membranes for effective oil-water separation, Nat. Commun., 2012, 3, 1025.

4 W. Zhang, Y. Zhu, X. Liu, D. Wang, J. Li, L. Jiang and J. Jin, Salt-induced fabrication of superhydrophilic and underwater superoleophobic PAA-g-PVDF membranes for effective separation of oil-in-water emulsions, Angew. Chem., Int. Ed., 2014, 53, 856-860.

5 J. Zhang and S. Seeger, Polyester materials with superwetting silicone nanofilaments for oil/water separation and selective oil absorption, Adv. Funct. Mater., 2011, 21, 4699-4704.

6 T.-T. Lim and X. Huang, In situ oil/water separation using hydrophobic-oleophilic fibrous wall: a lab-scale feasibility study for groundwater cleanup, J. Hazard. Mater., 2006, 137, 820-826.

7 J. Li, L. Yan, X. Tang, H. Feng, D. Hu and F. Zha, Robust Superhydrophobic Fabric Bag Filled with Polyurethane Sponges Used for Vacuum-Assisted Continuous and Ultrafast Absorption and Collection of Oils from Water, Adv. Mater. Interfaces, 2016, 3, 1500770.

8 L. Feng, Z. Zhang, Z. Mai, Y. Ma, B. Liu, L. Jiang and D. Zhu, A super-hydrophobic and super-oleophilic coating mesh film for the separation of oil and water, Angew. Chem., Int. Ed., 2004, 116, 2046-2048.

9 S. Gao, X. Dong, J. Huang, S. Li, Y. Li, Z. Chen and Y. Lai, Rational construction of highly transparent superhydrophobic coatings based on a non-particle, fluorine-free and water-rich system for versatile oil-water separation, Chem. Eng. J., 2018, 333, 621-629.

10 X. J. Feng and L. Jiang, Design and creation of superwetting/ antiwetting surfaces, Adv. Mater., 2006, 18, 3063-3078.

11 J. He, W. Ji, L. Yao, Y. Wang, B. Khezri, R. D. Webster and H. Chen, Strategy for Nano-Catalysis in a Fixed-Bed System, Adv. Mater., 2014, 26, 4151-4155.

$12 \mathrm{~K}$. Liu and L. Jiang, Metallic surfaces with special wettability, Nanoscale, 2011, 3, 825-838.

13 W. Zhang, Z. Shi, F. Zhang, X. Liu, J. Jin and L. Jiang, Superhydrophobic and superoleophilic PVDF membranes for effective separation of water-in-oil emulsions with high flux, Adv. Mater., 2013, 25, 2071-2076.

14 S. Pan, A. K. Kota, J. M. Mabry and A. Tuteja, Superomniphobic surfaces for effective chemical shielding, J. Am. Chem. Soc., 2013, 135, 578-581.
15 L. Aurelie and Q. David, Superhydrophobic states, Nat. Mater., 2003, 2, 457.

16 B. Wang, W. X. Liang, Z. G. Guo and W. M. Liu, Biomimetic super-lyophobic and super-lyophilic materials applied for oil/water separation: a new strategy beyond nature, Chem. Soc. Rev., 2015, 44, 336-361.

17 Y. Xin and Z. G. Guo, Robust superhydrophobic zinc oxide film, Chem. Lett., 2013, 43, 305-306.

18 (a) Y. Shen, Z. G. Guo and W. M. Liu, Biomimetic transparent and superhydrophobic coatings: from nature and beyond nature, Chem. Commun., 2015, 51, 1775-1794; (b) J. C. Love, B. D. Gates, D. B. Wolfe, K. E. Paul and G. M. Whitesides, Fabrication and wetting properties of metallic half-shells with submicron diameters, Nano Lett., 2002, 2, 891-894.

19 (a) M. Jin, X. Feng, L. Feng, T. Sun, J. Zhai, T. Li and L. Jiang, Superhydrophobic aligned polystyrene nanotube films with high adhesive force, Adv. Mater., 2005, 17, 1977-1981; (b) F. Shi, J. Niu, J. Liu, F. Liu, Z. Wang, X. Q. Feng and $\mathrm{X}$. Zhang, Towards understanding why a superhydrophobic coating is needed by water striders, Adv. Mater., 2007, 19, 2257-2261.

20 (a) Z. G. Guo, W. M. Liu and B. L. Su, Superhydrophobic surfaces: from natural to biomimetic to functional, $J$. Colloid Interface Sci., 2011, 353, 335-355; (b) D. Han and A. J. Steckl, Superhydrophobic and oleophobic fibers by coaxial electrospinning, Langmuir, 2009, 25, 9454-9462.

21 (a) T. Jiang, Z. G. Guo and W. M. Liu, Biomimetic superoleophobic surfaces: focusing on their fabrication and applications, J. Mater. Chem. A, 2015, 3, 1811-1827; (b) X. Feng, L. Feng, M. Jin, J. Zhai, L. Jiang and D. Zhu, Reversible super-hydrophobicity to super-hydrophilicity transition of aligned $\mathrm{ZnO}$ nanorod films, J. Am. Chem. Soc., 2004, 126, 62-63.

22 (a) G. Y. Wang, Z. G. Guo and W. M. Liu, Interfacial effects of superhydrophobic plant surfaces: a review, J. Bionic. Eng., 2014, 11, 325-345; (b) D. Tian, Q. Chen, F. Q. Nie, J. Xu, Y. Song and L. Jiang, Patterned wettability transition by photoelectric cooperative and anisotropic wetting for liquid reprography, Adv. Mater., 2009, 21, 3744-3749.

23 (a) J. T. Han, D. H. Lee, C. Y. Ryu and K. Cho, Fabrication of superhydrophobic surface from a supramolecular organosilane with quadruple hydrogen bonding, J. Am. Chem. Soc., 2004, 126, 4796-4797; (b) J. Li, D. Li, Y. Yang, J. Li, F. Zha and Z. Lei, A prewetting induced underwater superoleophobic or underoil (super) hydrophobic waste potato residue-coated mesh for selective efficient oil/water separation, Green Chem., 2016, 18, 541-549.

24 (a) J. Wang, Y. Wen, J. Hu, Y. Song and L. Jiang, Fine Control of the Wettability Transition Temperature of Colloidal-Crystal Films: From Superhydrophilic to Superhydrophobic, Adv. Funct. Mater., 2007, 17, 219-225; (b) K. K. S. Lau, J. Bico, K. B. K. Teo, M. Chhowalla, G. A. J. Amaratunga, W. I. Milne, G. H. McKinley and K. K. Gleason, Superhydrophobic carbon nanotube forests, Nano Lett., 2003, 3, 1701-1705. 
25 A. Steele, I. Bayer and E. Loth, Inherently superoleophobic nanocomposite coatings by spray atomization, Nano Lett., 2008, 9, 501-505.

26 H. Alamri, A. Al-Shahrani, E. Bovero, T. Khaldi, G. Alabedi, W. Obaid and A. Fihri, Self-cleaning superhydrophobic epoxy coating based on fibrous silica-coated iron oxide magnetic nanoparticles, J. Colloid Interface Sci., 2018, 513, 349-356.

27 B. Cortese, D. Caschera, F. Federici, G. M. Ingo and G. Gigli, Superhydrophobic fabrics for oil-water separation through a diamond like carbon (DLC) coating, J. Mater. Chem. A, 2014, 2, 6781-6789.

28 X. Zhou, Z. Zhang, X. Xu, F. Guo, X. Zhu, X. Men and B. Ge, Robust and durable superhydrophobic cotton fabrics for oil/ water separation, ACS Appl. Mater. Interfaces, 2013, 5, 72087214.

29 S. Khorsand, K. Raeissi, F. Ashrafizadeh and M. A. Arenas, Super-hydrophobic nickel-cobalt alloy coating with micronano flower-like structure, Chem. Eng. J., 2015, 273, 638-646.

$30 \mathrm{~J}$. Zhang and S. Seeger, Polyester materials with superwetting silicone nanofilaments for oil/water separation and selective oil absorption, Adv. Funct. Mater., 2011, 21, 4699-4704.

31 F. Qiang, L. L. Hu, L. X. Gong, L. Zhao, S. N. Li and L. C. Tang, Facile synthesis of super-hydrophobic, electrically conductive and mechanically flexible functionalized graphene nanoribbon/polyurethane sponge for efficient oil/water separation at static and dynamic states, Chem. Eng. J., 2018, 334, 2154-2166.

32 M. Liu, Y. Hou, J. Li, L. Tie and Z. Guo, An all-water-based system for robust superhydrophobic surfaces, J. Colloid Interface Sci., 2018, 519, 130-136.

33 K. Feng, G. Y. Hung, J. Liu, M. Li, C. Zhou and M. Liu, Fabrication of high performance superhydrophobic coatings by spray-coating of polysiloxane modified halloysite nanotubes, Chem. Eng. J., 2018, 331, 744-754.

34 H. Zhou, H. Wang, H. Niu, A. Gestos and T. Lin, Robust, self-healing superamphiphobic fabrics prepared by two-step coating of fluoro-containing polymer, fluoroalkyl silane, and modified silica nanoparticles, Adv. Funct. Mater., 2013, 23, 1664-1670.
35 J. Li, C. Xu, C. Guo, H. Tian, F. Zha and L. Guo, Underoil superhydrophilic desert sand layer for efficient gravitydirected water-in-oil emulsions separation with high flux, $J$. Mater. Chem. A, 2018, 6, 223-230.

$36 \mathrm{D}$. Li and Z. Guo, Stable and self-healing superhydrophobic MnO2@ fabrics: Applications in self-cleaning, oil/water separation and wear resistance, J. Colloid Interface Sci., 2017, 503, 124-130.

37 J. Li, L. Shi, Y. Chen, Y. Zhang, Z. Guo, B.-L. Su and W. Liu, Stable superhydrophobic coatings from thiol-ligand nanocrystals and their application in oil/water separation, J. Mater. Chem., 2012, 22, 9774-9781.

38 W. Wang, S. J. You, X. B. Gong, D. P. Qi, B. K. Chandran, L. P Bi, F. Y. Cui and X. D. Chen, Bioinspired Nanosucker Array for Enhancing Bioelectricity Generation in Microbial Fuel Cells, Adv. Mater., 2016, 28, 270-275.

39 B. Wang, Y. Zhang, W. Liang, G. Wang, Z. Guo and W. Liu, A simple route to transform normal hydrophilic cloth into a superhydrophobic-superhydrophilic hybrid surface, $J$. Mater. Chem. A, 2014, 2, 7845-7852.

40 A. K. Sasmal, C. Mondal, A. K. Sinha, S. S. Gauri, J. Pal, T. Aditya, M. Ganguly, S. Dey and T. Pal, Fabrication of superhydrophobic copper surface on various substrates for roll-off, self-cleaning, and water/oil separation, ACS Appl. Mater. Interfaces, 2014, 6, 22034-22043.

41 J. Gao, X. Huang, H. Xue, L. Tang and R. K. Li, Facile preparation of hybrid microspheres for super-hydrophobic coating and oil-water separation, Chem. Eng. J., 2017, 326, 443-453.

42 I. E. Palamà, S. D'Amone, V. Arcadio, D. Caschera, R. G. Toro, G. Gigli and B. Cortese, Underwater Wenzel and Cassie oleophobic behaviour, J. Mater. Chem. A, 2015, 3, 3854-3861.

43 W. Ma, J. Zhao, O. Oderinde, J. Han, Z. Liu, B. Gao and C. Huang, Durable superhydrophobic and superoleophilic electrospun nanofibrous membrane for oil-water emulsion separation, J. Colloid Interface Sci., 2018, 532, 12-23.

44 Z. H. Zhang, R. B. Dua, L. B. Zhang, H. B. Zhu, H. N. Zhang and P. Wang, Carbon-Layer-Protected Cuprous Oxide Nanowire Arrays for Efficient Water Reduction, ACS Nano, 2013, 7, 1709-1717. 